\title{
Optically Induced Modulation of a Laser Beam in Nematic Liquid Crystals Structures ${ }^{\dagger}$
}

\author{
J.A. MARTIN-PEREDA, F.J. LOPEZ, and M. A. MURIEL
}

Departamento de Electronica Cuantica, E. T. S. Ing. Telecomunicacion, Universidad Politecnica de Madrid, Ciudad Universitaria, Madrid-3, Spain

In this paper we report the experimental results obtained when an He-Ne laser beam crosses an MBBA homeotropic sandwich structure and is modulated by the influence of another laser beam, in our case an $\mathrm{Ar}^{+}$laser, crossing through the same region. We extend some results previously reported by us $^{12}$ concerning the influence of the ratio of the diameters of the laser beams on the modulation characteristics.

A theoretical model, based on the one reported in $\operatorname{Ref}^{6}$, shows good agreement with the experimental results. If the $\mathrm{Ar}^{+}$laser is intensity chopped, the resulting $\mathrm{He}-\mathrm{Ne}$ diffracted image is also intensity modulated. The highest frequency observed has been 500 p.p.s.

\section{INTRODUCTION}

It was recently established, both theoretically ${ }^{1}$ and experimentally, ${ }^{2,3,4}$ that nematic liquid crystals can have large optical nonlinearities. This effect comes from the optical-field-induced, collective reorientation of molecules. A C. W. laser can be intense enough to yield a significant refractive index change in the medium.

Effects such as self-focusing and self-phase modulation have been the object of several papers treating nematic liquid crystals as non-linear materials. ${ }^{5-9}$ Studies have shown that the intensity-dependent refractive index associated with optically induced molecular reorientation in a typical nematic crystal, such as $p$-methoxybenzylidene- $p^{\prime}$-n-butylaniline (MBBA), is

\footnotetext{
${ }^{\dagger}$ Presented at the Ninth International Liquid Crystal Conference, Bangalore, December 6-10, 1982.
} 
extremely large. Third-order nonlinear optical processes, such as degenerate four-wave mixing and wave-front conjugation, are easily observable with lasers of moderate power (intensity of the order or $10 \mathrm{w} / \mathrm{cm}^{2}$ ). ${ }^{4,10}$ Two groups have recently published their observation of optical-field-induced effects in the nematic phase. Because of the large nonlinearity, selffocusing of the laser beam in these experiments was observed. ${ }^{1}$ In every case, the beam characteristics change drastically when the beams pass through even a thin sample of such materials. Some research has been done in this area. Shen et al ${ }^{6}$ have reported the observation of a multiple-ring pattern of laser diffraction from a nematic film. The phenomenon is shown to be the result of spatial, self-phase modulation that is due to the laserinduced Fréedericksz transition. Moreover, it has been shown that the dynamic behavior of this optical-field-induced transition is also analogous to that of the magnetically induced case. ${ }^{5}$ The initial response of the induced molecular reorientation to the laser switch-on and the larger time response to laser switch-off are both exponential with the relaxation times $\tau_{\text {on }}$ and $\tau_{\text {off. }}$ The values given by Shen et al. working at an $\mathrm{Ar}^{+}$-laser intensity of $350 \mathrm{w} / \mathrm{cm}^{2}$ are close to $100 \mathrm{sec}$, showing good agreement with their theory. They used a He-Ne laser to prove the optical birefringence resulting from the induced molecular reorientation.

In this paper we extend some results reported elsewhere by us $^{11-13}$ concerning qualitative and quantitative measurements of the optically induced modulation of a laser beam in nematic liquid crystal (N.L.C.) structures by another laser employed as a modulator.

\section{EXPERIMENTAL}

The experiment uses a C. W. Ar ion laser focused to an $e^{-2}$ diameter of around $25 \mu \mathrm{m}$ at the homeotropically aligned cell depicted in Figure 1. A thin film of MBBA was employed as the nonlinear material. The optical propagation is at an angle of $0^{\circ}$ with the liquid crystal molecular axis.

A second, He-Ne laser beam makes an angle of about $40^{\circ}$ with respect to the $\mathrm{Ar}^{+}$laser. Another lens was used to position this second laser beam while controlling its spot size on the sample. The relative beam-diameters at the liquid-crystal cell are given by:

$$
w / a \simeq\left(f_{2} \lambda_{2} w_{1}\right) /\left(f_{1} \lambda_{1} w_{1}\right)+m\left(\pi w_{1} w_{2}\right) /\left(f_{1} f_{2} \lambda_{1}\right)
$$

where $f_{1}$ and $f_{2}$ are the focal lengths of the lenses focusing the $\mathrm{Ar}$ and the He-Ne lasers, respectively, and $m$ is the distance between the focus position of lens $f_{2}$ and the middle of the liquid-crystal cell. $\lambda_{1}$ and $\lambda_{2}$ are the $\mathrm{Ar}^{+}$and He-Ne laser-beam radii, respectively. 


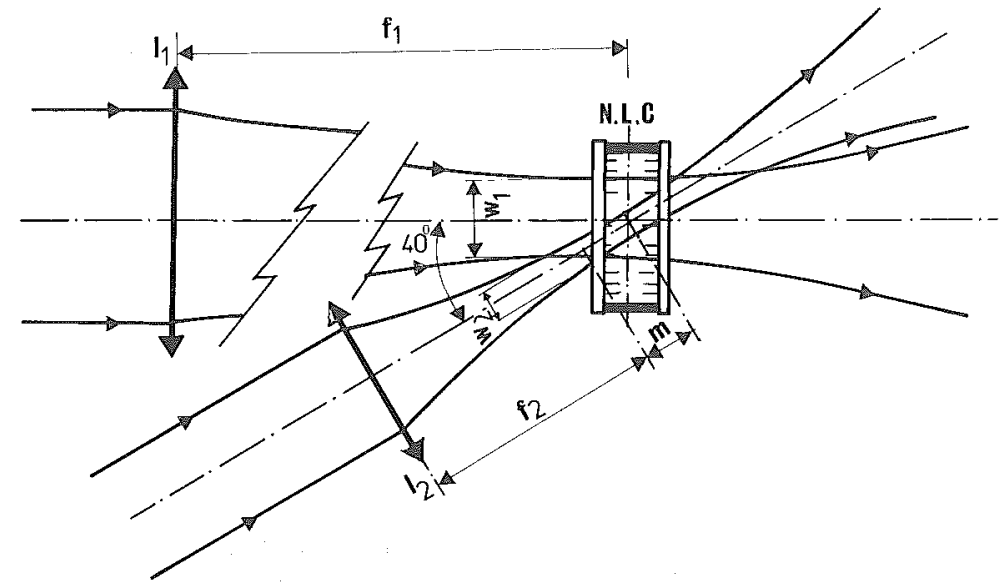

FIGURE 1 Experimental set-up. NLC, homeotropic nematic liquid-crystal cell. Other symbols are defined in the text. $f_{1}>f_{2}$.

In our experimental set-up, Eq. 1 is therefore

$$
w / a=0.5+3.45 m
$$

Both lasers, $5145 \AA$ and $6328 \AA \mathrm{He}-\mathrm{Ne}$, are linearly polarized with the same polarization direction, which is orthogonal to the plane containing both beams. The maximum output power for the $\mathrm{Ar}^{+}$and He-Ne lasers was 25 and $1 \mathrm{~mW}$, respectively.

The samples studied have a separation between plates smaller than $120 \mu \mathrm{m}$. The homeotropic alignment was obtained by surface treatment using hexadecyltrimethyl ammonium bromide (HTAB). The experiments were carried out at room temperature (about $25^{\circ} \mathrm{C}$ ), i.e., within the nematic range of MBBA.

We projected both laser beams onto the same screen, which was situated about $1 \mathrm{~m}$ from the nematic cell. A photodetector was placed at the middle of the He-Ne image to register the intensity variations.

When no $\mathrm{Ar}^{+}$laser was applied to the liquid-crystal cell, the projected He-Ne laser beam had the usual Gaussian intensity profile, as could be observed by a transverse displacement of the photodetector. The same situation was obtained when the $\mathrm{Ar}^{+}$laser was turned on, as long as power densities, on the cell, were smaller than threshold intensity (about $1 \mathrm{~kW} / \mathrm{cm}^{2}$ depending upon sample thickness). Larger powers for the $\mathrm{Ar}^{+}$laser gave rise to self-focusing as had been shown by Khoo. ${ }^{1}$ The divergence of the $\mathrm{Ar}^{+}$beam after crossing the sample progressively decreases as the intensity is increased, and concentric rings begin to appear. Recent studies have shown that these rings are due to the large phase 
modulation of the laser owing to the birefringence of the tilted molecules. The appearance of these rings denotes that a large molecular reorientation has occurred.

If the focused He-Ne laser beam now crosses the self-focused zone, its focus being at a distance between $5 \mathrm{~mm}$ and $3 \mathrm{~cm}$ from the middle of the liquid crystal cell, a different intensity profile is obtained at the screen. If the He-Ne laser is not focused, after crossing the optically reoriented zone, a different intensity profile is observed, similar to the diffraction pattern of a circular black spot (in this case, another lens was placed behind the cell).

Depending on the $\mathrm{Ar}^{+}$laser intensity, different intensity profiles can be obtained for the focused He-Ne laser beam. The results are shown in Figure 2 where the intensity given by the photodetector is represented as a

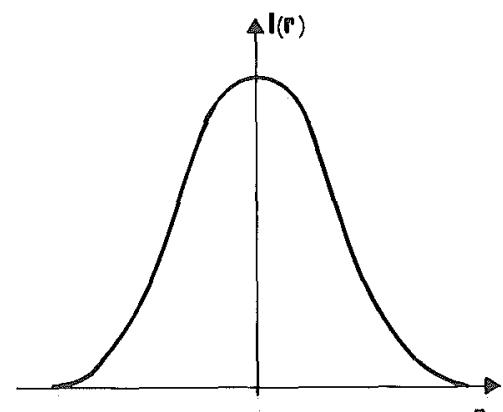

a)

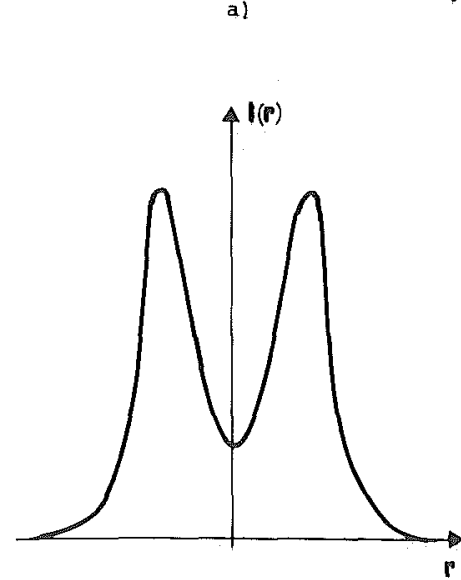

c)
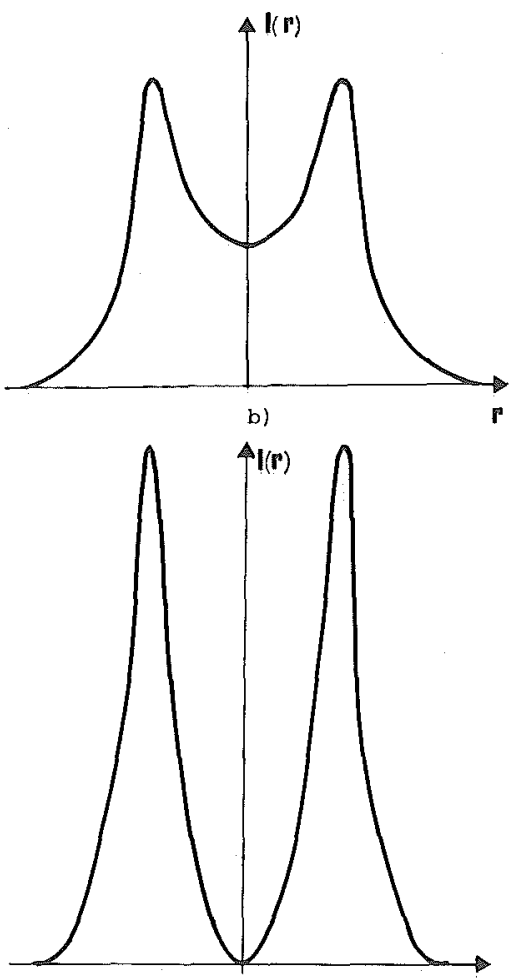

d)

FIGURE 2 Transverse profile of He-Ne beam at the screen, for increasing modulator optical intensity.
(a) $I_{\mathrm{Ar}}+=0$
(b) $I_{\mathrm{Ar}^{+}}=1.5 I_{\mathrm{th}}$
(c) $I_{\mathrm{Ar}}+=3 I_{\mathrm{th}}$
(d) $I_{\mathrm{Ar}}+\geqslant I_{\mathrm{th}}$ 
function of the distance from the center of the diffraction figure. These graphics have been normalized and symmetrized to compensate for possible photodetector misalignments. The first one (2a) corresponds to no $\mathrm{Ar}^{+}$laser and gives the usual Gaussian profile. In the other three are shown the results for increasing power in the $\mathrm{Ar}^{+}$laser. As can be seen, the initial Gaussian profile no longer appears, and in the last case, a small bright ring appears around a dark center. In Figure 3, the variation of the light intensity in the center of the He-Ne laser is represented as a function of the $\mathrm{Ar}^{+}$ion intensity.

When the $\mathrm{Ar}^{+}$intensity reaches a value about $3 \mathrm{~kW} / \mathrm{cm}^{2}$, rings such as the ones reported by Shen appear in the $\mathrm{Ar}^{+}$pattern. A similar effect occurs with the He-Ne laser but with a much smaller contrast between maxima and minima. The same black dot remains at the center of the projected image. The energy has been transferred from the initial Gaussian profile to the surrounding ring.

\section{THEORETICAL MODEL}

An important point that needs to be considered is the relative size at the cell interaction zone of the two propagating laser beams. The phenomenon described above, i.e., a black spot at the center of the emerging He-Ne beam, appears at a given diameter ratio between the $\mathrm{Ar}^{+}$and the $\mathrm{He}-\mathrm{Ne}$ beams. Other ratios give a different diffraction image. This result can be described by a model similar to the one employed previously ${ }^{6}$ to describe

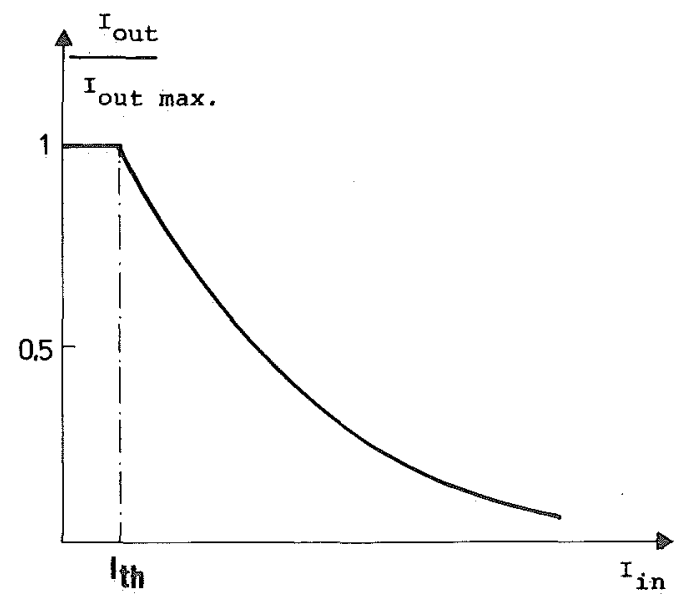

FIGURE 3 Transference function of modulator systems. $I_{\text {out }}$ is measured at the center of the He-Ne beam for a ratio $w / a=0.7$ between spot sizes at the cell. 
the laser-induced diffraction rings from a N. L.C. film. We can describe both lasers, at entrance to the cell, by the following equations:

$$
\begin{aligned}
& E_{\mathrm{s}}(\rho, 0)=E_{0} \exp \left(-\rho^{2} / a^{2}\right) \\
& E_{\mathrm{w}}(\rho, 0)=E_{\mathrm{l}} \exp \left(-\rho^{2} / w^{2}\right)
\end{aligned}
$$

where $a$ and $w$ are the $\mathrm{Ar}^{+}$- and the He-Ne laser-waist radii respectively, as before. Above a certain threshold, the $\mathrm{Ar}^{+}$-laser field $E_{\mathrm{s}}$ will reorient the direction of the molecular alignment through the medium. This leads to a local refractive-index change $\Delta n(\rho)$ in the medium seen by both lasers and to two corresponding phase shifts. The new refractive index will be given by

$$
n(\rho)=n_{0}+n_{2} E_{0}^{2} \exp \left(-2 \rho^{2} / a^{2}\right)
$$

Therefore the He-Ne-laser beam suffers a phase retardation given by:

$$
\psi(\rho)=\frac{2 \pi d}{\lambda_{2}} n(\rho)=\psi_{0}+\psi_{1}(\rho)
$$

with

$$
\psi_{0}=\frac{2 \pi d n_{0}}{\lambda_{2}} \quad \text { and } \quad \psi_{1}=\phi_{1} \exp \left(-\frac{2 \rho^{2}}{a^{2}}\right)
$$

where $d$ is the sample thickness and $\psi_{0}$ is the phase retardation with no optical-induced reorientation of the molecular alignment.

The outgoing field for the He-Ne-laser beam after it crosses the liquidcrystal cell will be given, therefore by:

$$
E_{\mathrm{w}}(\rho)=\frac{1}{2}\left\{E_{0} \exp \left(-\frac{\rho^{2}}{w^{2}}\right) \cdot \exp \left[-i \phi_{1} \exp \left(-\frac{2 \rho^{2}}{a^{2}}\right)\right]+\text { c.c. }\right\}
$$

Owning to the small diameter of the interaction zone, we can use the far field approximation (Fraunhofer) for cell-to-screen distances larger than several centimeters. The intensity on the screen will be:

$$
I\left(r ; \phi_{1} ; a, w\right)=|F(r ; \phi ; a ; w)|^{2}
$$

where:

$$
\begin{aligned}
& F\left(r ; \phi_{1} ; a, w\right)=2 \pi \int_{0}^{\infty} \rho E_{0} \exp \left(-\frac{\rho^{2}}{w^{2}}\right) \\
& \cdot \exp \left[-i \phi_{1} \exp \left(-\frac{2 \rho^{2}}{a^{2}}\right)\right] \cdot J_{0}(2 \pi r \rho) \mathrm{d} \rho
\end{aligned}
$$

This integral can be expressed, after some manipulations, as: 

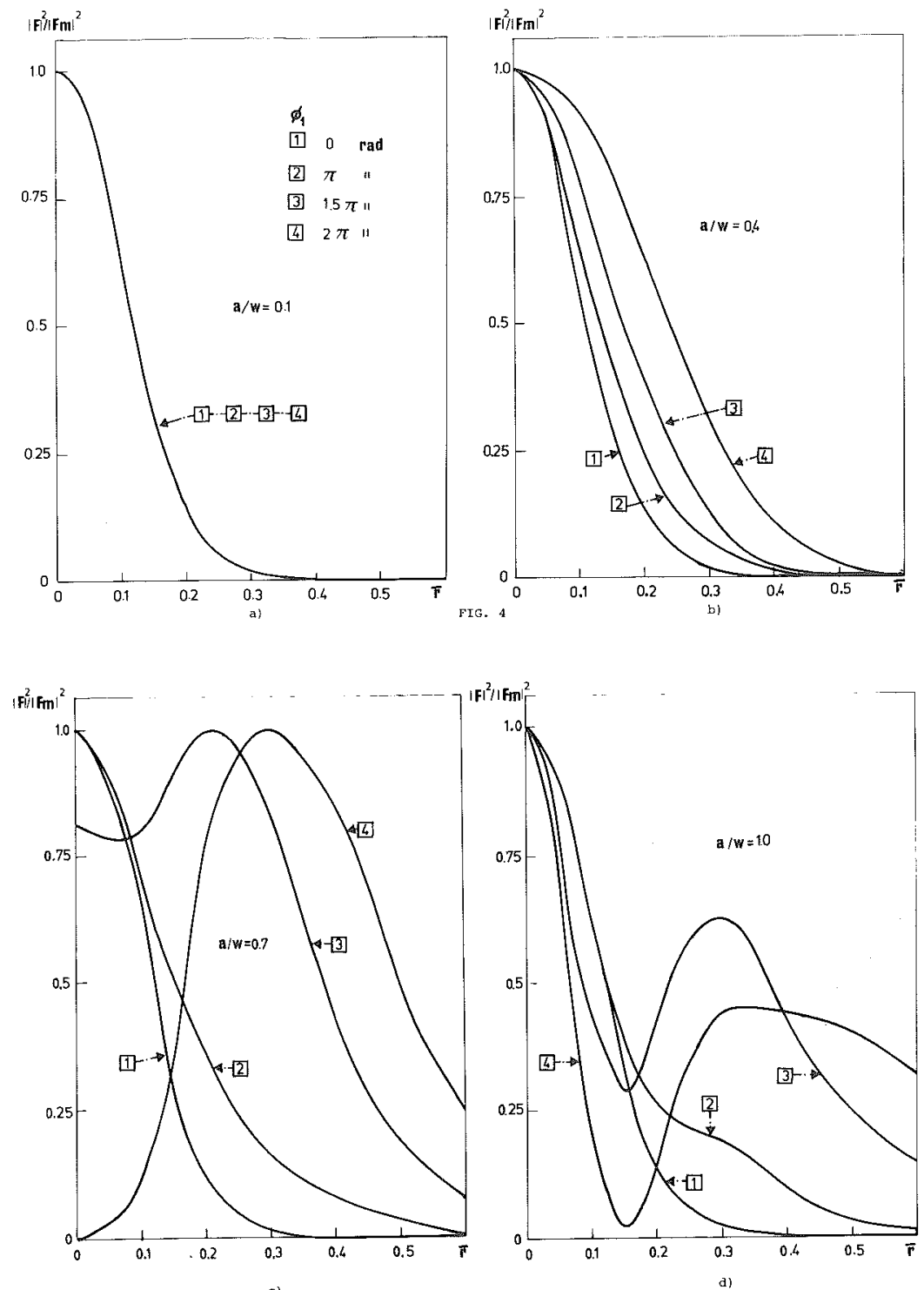

FIGURE 4 Calculated diffracted wave fields for the He-Ne laser, different intensity values for the modulating $\mathrm{Ar}^{+}$laser, and different relative spot diameters. $\emptyset_{1}$ and $\bar{r}$ are defined in the text. 
TABLE I

Response times of the output signal as a function of $\mathrm{Ar}^{+}$-laser intensities, sample thickness and focal distance of the $\mathrm{Ar}^{+}$input chopped beam

\begin{tabular}{ccccc}
\hline$d(\mu \mathrm{m})$ & $I\left(\mathrm{~kW} / \mathrm{cm}^{2}\right)$ & $\tau_{1}(\mathrm{~ms})$ & $\tau_{2}(\mathrm{~ms})$ & $f\left(\mathrm{~L}_{1}\right)$ \\
\hline \multirow{2}{*}{125} & 3.26 & 28 & 24 & $7 \mathrm{~cm}$ \\
& 1.91 & 28 & 32 & \\
\multirow{4}{*}{100} & 1.47 & 28 & 34 & \\
& 3.26 & 22 & 18 & \\
& 1.91 & 22 & 20 & \\
125 & 1.47 & 22 & 22 & $6.7 \mathrm{~cm}$ \\
& 3.56 & 10 & 9 & \\
& 2.09 & 10 & 11.5 & \\
100 & 1.35 & 10 & 12 & \\
& 3.56 & 8 & 7.5 & \\
& 2.09 & 8 & 8.2 & \\
& 1.35 & 8 & 8.6 & \\
\hline
\end{tabular}

$$
F\left(r ; \phi_{1} ; a / w\right)=\int_{0}^{1} \exp \left[i \phi_{1} t^{-2(a / w)^{2}}\right] J_{0}[\bar{r} \sqrt{\ln (1 / t)}] \mathrm{d} t
$$

where

$$
\bar{r}=2 \pi a r
$$

As can be seen this diffraction pattern does not depend upon $a$ and $w$ independently, but upon the ratio, $a / w$.

Figure 4 is a plot of the numerical solution of Eq. 11 for several values of $\phi_{1}$ and $a / w$ parameters.

This diffraction pattern is pictured in Figure 4 for $w / a=0.1,0.4,0.7$, and 1.0 and for different values of $\phi_{1}$. In each case represented, the maximum value has an assigned value of unity.

\section{DYNAMICAL BEHAVIOR OF THE SYSTEM}

To observe the dynamical behavior of this phenomenon, a chopper was placed in the $\mathrm{Ar}^{+}$-laser beam before the beam crossed the liquid crystal cell; a photodetector was situated at the center of the diffracted He-Nelaser beam.

The main results are shown in Table I where response times of the output signal are given as a function of $\mathrm{Ar}^{+}$-laser intensities, sample thickness, and focal distance of the $\mathrm{Ar}^{+}$input-chopped beam $\left(f_{1}\right)$. Two response times are given: the first one corresponds to the turn on and the second one to 
the turn off of the molecular. Orientation from these data, the cut-off frequency of the system will be around 25 p.p.s. This value is better than experimental results previously published by us. ${ }^{12} \mathrm{~A}$ further point needs to be considered, namely, that lens focal distance has a greater importance than cell thickness.

\section{CONCLUSIONS}

We have shown that the optically induced modulation of a laser beam in N. L. C. structures by another beam laser, employed as a modulator, is possible. Some modifications concerning cell structure and focusing parameters need to be taken into account and will be published shortly.

\section{Acknowledgment}

We thank J.M. Oton for sample fabrication and MBBA synthesis. This work was partly supported by the Spanish "Comisión Asesora de Investigación Científica y Técnica" (Grant 3864-79).

\section{References}

1. I. C. Khoo, S. L. Zhuang and S. Shepard, Appl. Phys. Lett., 39, 937 (1981).

2. I. C. Khoo and S. L. Zhuang, Appl. Phys. Lett., 37, 3 (1980).

3. I. C. Khoo, Phys. Rev., A23, 2077 (1981).

4. I. C. Khoo, Phys. Rev., A25, 1040, 1636 (1982).

5. S. D. Durbin, S. M. Arakelian and Y. R. Shen, Phys. Rev. Lett., 47, 1411 (1981).

6. S. D. Durbin, S. M. Arakelian and Y. R. Shen, Opt. Lett., 6, 411 (1981).

7. A. S. Zolot'ko, V. F. Kitaeva, N. Kroo, N. N. Sobolev and L. Csillag, JETP Lett., 32, $158(1980)$.

8. B. Ya Zel'dovich, N. F. Pilipetskii, A. V. Sukhov and N. V. Tabiryan, JETP Lett., 31, $263(1980)$.

9. N. Kroo, N. N. Sobolev and L. Chillag, JETP Lett., 32, 158, (1980).

10. R. M. Herman and R. J. Sherinko, Phys, Rev., A19, 1757 (1979).

11. J. A. Martin-Pereda and F. J. López, in Digest of Conference on Lasers and ElectroOptics (Optical Society of America, Washington, D.C., 1982), paper WC14.

12. J. A. Martin-Pereda and F. J. López, Opt. Lett., 7, 590 (1982).

13. J. A. Martin-Pereda and F. J. López, "Optically induced modulation of a laser beam in nematic liquid crystal structures." Also presented at the Ninth International Liquid Crystal Conference, Bangalore, December 6-10, 1982. 